\title{
Myxoma of the upper leg originating from an appendiceal mucinous neoplasm: A case report
}

\author{
JUN LI $^{1}$, XUE-YAN ZHANG ${ }^{2}$, BIN WANG $^{3}$ and QING-YONG CAO ${ }^{1}$ \\ ${ }^{1}$ Department of Radiology, Yantai Affiliated Hospital of Binzhou Medical University, Yantai, Shandong 264100; \\ ${ }^{2}$ Nursing College; ${ }^{3}$ Medical Imaging Research Institute, Binzhou Medical University, Yantai, Shandong 264003, P.R. China
}

Received October 4, 2014; Accepted July 7, 2015

DOI: $10.3892 / \mathrm{ol} .2015 .3582$

\begin{abstract}
Myxoma is rare in the upper leg. The current study presents the case of a large tumor in the right upper leg. The tumor extended to pelvic cavity and was found to be connected with a cystic and solid neoplasm that was adjacent to the ascending colon in the right lower quadrant. The large tumor of the upper leg had existed for 15 years and had ulcerated through the skin 4 days prior to admittance. Palliative surgery was performed, with histological findings of a myxoma. Since appendiceal mucinous neoplasms may invade organizations outside of the mucous layer of the appendix and cause secondary peritoneal myxoma, this myxoma of the upper leg probably originated from an appendiceal mucinous neoplasm.
\end{abstract}

\section{Introduction}

Myxomas are benign neoplasms that are characterized by slow growth (1). The most frequent location of those neoplasms is the cardiac muscle, where they account for $50 \%$ of all benign lesions (2). Myxoma is rare in the leg, but can occur within its muscles (1). To date, $<10$ cases of myxoma located in the leg have been documented (1,3-5). Computed tomography usually shows a well-delimited mass with an absorption density between that of muscle and water. The most precise examination technique that enables diagnosis is magnetic resonance (MR) imaging $(6,7)$, and radical removal of the whole lesion is the most effective method of treating myxomas (8). The present study reports a case of myxoma localized to the leg and interlinked with the pelvic cavity, which was treated with palliative surgery. To the best of our knowledge, this study is the first to describe the possibility of a leg myxoma originating from an appendiceal mucinous neoplasm.

Correspondence to: Mr. Jun Li, Department of Radiology, Yantai Affiliated Hospital of Binzhou Medical University, 717 Jin-Bu Street, Yantai, Shandong 264100, P.R. China

E-mail: bzmceducn@sina.com

Key words: myxoma, upper leg, appendix, neoplasm

\section{Case report}

An 88-year-old female was hospitalized at Yantai Affiliated Hospital of Binzhou Medical University (Yantai, China) in January 2014 with a large tumor in the right upper leg. The tumor had existed for 15 years and had ulcerated through the skin 4 days prior to admittance. The function and appearance of the right lower limb were seriously affected. Laboratory examinations showed no abnormalities and no other symptoms were observed. The MR studies were performed by a SIEMENS Avanto 1.5T MR scanner. A large tumor $(45 \times 15 \times 20 \mathrm{~cm})$ in the right thigh was found with a slight high signal on T1-weighted imaging (Fig. 1A) and an appreciable high signal on T2-weighted imaging (Fig. 1B). The tumor extended to the pelvic cavity and was found to be connected with a cystic and solid neoplasm (Fig. 1C). Due to the older age of the patient, total resection was considered to be too traumatic. Instead, the patient underwent palliative surgery for the tumor in the upper leg, and the tumor was found to be full of brown and yellow gelatinous material. Myxoma was finally diagnosed via histological examination (Fig. 2). Following surgery, the patient recovered well and was subsequently discharged. The patient rejected further treatment and no follow-up examination was planned. At the time of writing, the patient was alive.

This study was approved by the Ethics Committee of Binzhou Medical University (Yantai, Shandong, China) and written informed consent was obtained from the patient.

\section{Discussion}

Myxoma is rare in the upper leg (3). Similarly to a previous study (1), the growth of the myxoma in the present case was slow. A plain X-ray may be normal or rarely visualize the lesion within the soft tissues as shading with calcifications inside (9). An ultrasonographic examination usually visualizes hypoechogenic lesions with fluid compartments located within the muscles. Computed tomography usually shows a well-delimited mass with an absorption density between that of muscle and water. The most precise examination technique that enables diagnosis is magnetic resonance imaging $(6,7)$.

Similarly to previous studies $(1,6,9)$, the myxoma in the present study had a sharply defined border. In addition, it exhibited a signal intensity lower than that of the skeletal 
A

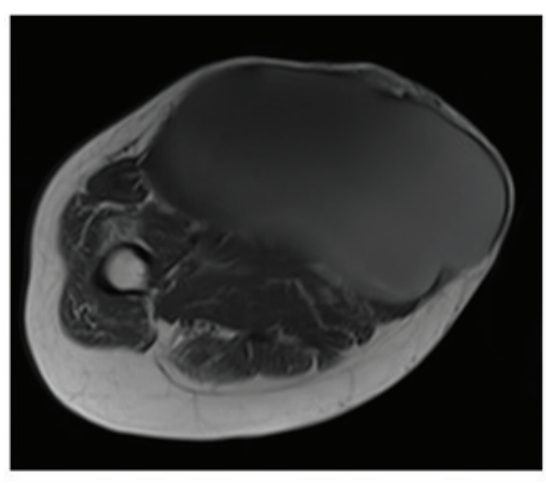

B

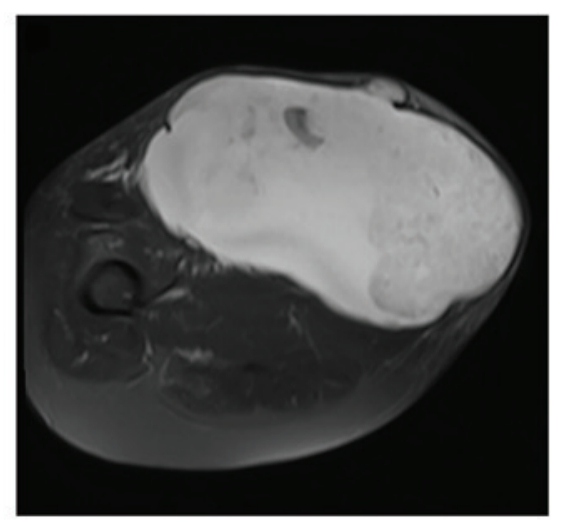

C

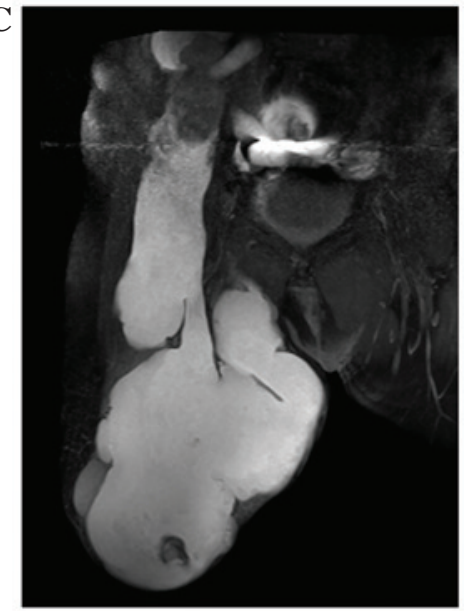

Figure 1. (A) Axial T1-weighted image showing the large mass of the right thigh with a slightly high signal. (B) Axial fat-supression T2-weighted image showing the large mass of the right thigh with an appreciable high signal. (C) Coronal Fat-supression T2-weighted image showing the assciation between the mass and the pelvic cavity; the mass extended to a cystic and solid neoplasm in the right lower quadrant.

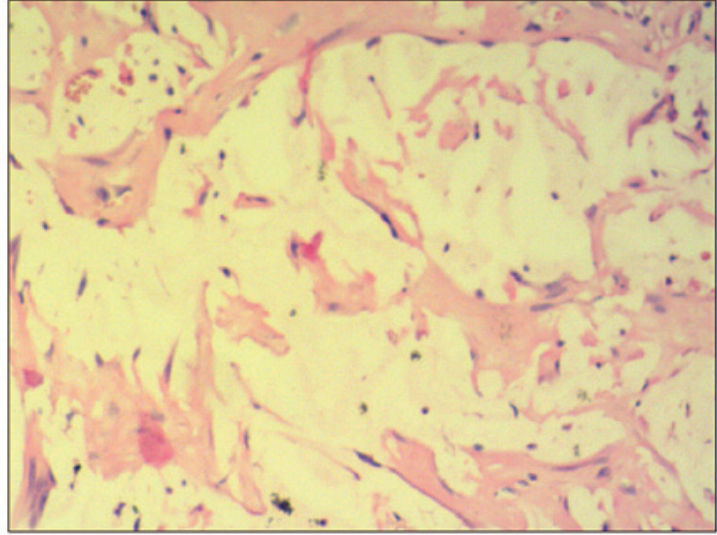

Figure 2. Histological findings of myxoma. The tumor was composed of bland spindle- and stellate-shaped cells in an abundant myxoid stroma.

muscles on T1-weighted images and brighter than adipose tissue on T2-weighted images.

In the present case, the large tumor of the thigh was interlinked with the pelvic cavity. It is important to observe the association between the lesion and pelvic organs, such as the appendix or ovaries, as appendiceal mucinous neoplasm may invade organizations outside of the mucous layer of the appendix and cause secondary peritoneal myxoma (10). Inside the mucus, epithelial cells rich in secretory functions can be found (11). In the present case, no epithelial cells were found through pathological examination. The reason for this may be associated with an inadequate local palliative surgery.

The patient rejected further treatment and no follow-up examination was planned. Since the tumor extended to the pelvic cavity and was found to be connected with a cystic and solid neoplasm that was adjacent to the ascending colon in the right lower quadrant, this case of myxoma probably originated from an appendiceal mucinous neoplasm.
In conclusion, the present study showed that the growth of myxoma is slow, with a long disease course. Furthermore, appendiceal mucinous neoplasms may invade outside of the mucosal layer of the appendix, leading to secondary myxoma, which may extend to the leg, as observed in the present study.

\section{References}

1. Spychała A, Murawa D and Niziołek A: Intramuscular myxoma of the left leg - Case report of the lesion observed for several years. Rep Pract Oncol Radiother 16: 71-74, 2011.

2. Reynen K: Cardiac myxomas. N Engl J Med 333: 1610-1617, 1995.

3. Luna A, Martinez S and Bossen E: Magnetic resonance imaging of intramuscular myxoma with histological comparison and a review of the literature. Skeletal Radiol 34: 19-28, 2005.

4. Ganchev G and Kunev S: A case of myxoma of the left leg with malignant clinical course. Khirurgiia (Sofiia) 20: 166-168, 1967 (In Bulgarian).

5. Avninder S, Ramesh V and Vermani S: Benign nerve sheath myxoma (myxoid neurothekeoma) in the leg. Dermatol Online J 13: 14, 2007.

6. Murawa D, Gowin E, Pawelska A and Murawa P: Cases of giant retroperitoneal liposarcomas. Rep Pract Oncol Radiother 10: 147-151, 2005.

7. Van Roggen J, McMenamin M and Fletcher D: Cellular myxoma of soft tissue: A clinicopathologic study of 38 cases confirming indolent clinical behaviour. Histopathology 39: 287-297, 2001.

8. Luebke AM, Gocke C, Priemel M, Grob TJ and Zustin J: Intramuscular myxoma of the lower leg. Pathologe 34: 360-363, 2013 (In German).

9. McCook T, Martinez S, Korobkin M, Ram PC, Bowen JH, Breiman RS, Harrelson JM and Gehweiler JA Jr: Intramuscular myxoma. Radiographic and computed tomographic findings with pathologic correlation. Skeletal Radiol 7: 15-19, 1981.

10. Honnef I, Moschopulos M and Roeren T: Appendiceal mucinous cystadenoma. Radiographics 28: 1524-1527, 2008.

11. Caracappa D, Gullà N, Gentile D, Listorti C, Boselli C, Cirrochi R, Bellezza G and Noya G: Appendiceal mucocele. A case report and literature review. Ann Ital Chir 82: 239-245, 2011. 